\title{
Supplementary Material for Real-Time Bayesian Parameter Estimation for Item Response Models
}

\author{
Ruby Chiu-Hsing Weng ${ }^{1}$ and D. Stephen Coad $^{2}$ \\ National Chengchi University, Taipei, Taiwan ${ }^{1}$ \\ Queen Mary, University of London, $\mathrm{UK}^{2}$
}

\section{Appendix A}

This Appendix presents derivations of the update equations (26)-(28) in Section 4.1 of the main paper. Let $\phi$ and $\Phi$ denote the density and distribution function of $N(0,1)$, and let $\phi(x \mid \mu, \sigma)$ denote the density of $N\left(\mu, \sigma^{2}\right)$. Take the following three steps.

Step 1. Let

$$
f\left(\alpha_{j}^{*}, \beta_{j}^{*}, \theta_{i}^{*}\right)=\Phi\left(\beta_{j} \theta_{i}+\alpha_{j}-\gamma_{c-1}\right)-\Phi\left(\beta_{j} \theta_{i}+\alpha_{j}-\gamma_{c}\right),
$$

as in $(25)$ of the main paper. Obtain the following derivatives of $f$ :

$$
\begin{gathered}
\frac{\partial \log f}{\partial \alpha_{j}^{*}}=\sigma_{\alpha_{j}} \cdot \Omega(x, a), \quad \frac{\partial \log f}{\partial \beta_{j}^{*}}=\sigma_{\beta_{j}} \theta_{i} \cdot \Omega(x, a), \quad \frac{\partial \log f}{\partial \theta_{i}^{*}}=\sigma_{\theta_{i}} \beta_{j} \cdot \Omega(x, a), \\
\frac{\partial^{2} \log f}{\partial\left(\alpha_{j}^{*}\right)^{2}}=\sigma_{\alpha_{j}}^{2} \cdot \Delta(x, a), \frac{\partial^{2} \log f}{\partial\left(\beta_{j}^{*}\right)^{2}}=\left(\sigma_{\beta_{j}} \theta_{i}\right)^{2} \cdot \Delta(x, a), \frac{\partial^{2} \log f}{\partial\left(\theta_{r}^{*}\right)^{2}}=\left(\sigma_{\theta_{i}} \beta_{j}\right)^{2} \cdot \Delta(x, a), \\
\frac{\partial^{2} f / \partial\left(\alpha_{j}^{*}\right)^{2}}{f}=\sigma_{\alpha_{j}}^{2} \cdot\left(\frac{(x-a) \phi(x-a)-x \phi(x)}{\Phi(x)-\Phi(x-a)}\right)=\sigma_{\alpha_{j}}^{2} \cdot\left((\Omega(x, a))^{2}-\Delta(x, a)\right), \\
\frac{\partial^{2} f / \partial\left(\beta_{j}^{*}\right)^{2}}{f}=\left(\sigma_{\beta_{j}} \theta_{i}\right)^{2} \cdot\left(\frac{(x-a) \phi(x-a)-x \phi(x)}{\Phi(x)-\Phi(x-a)}\right)=\left(\sigma_{\beta_{j}} \theta_{i}\right)^{2} \cdot\left((\Omega(x, a))^{2}-\Delta(x, a)\right), \\
\frac{\partial^{2} f / \partial\left(\theta_{r}^{*}\right)^{2}}{f}=\left(\sigma_{\theta_{i}} \beta_{j}\right)^{2} \cdot\left(\frac{(x-a) \phi(x-a)-x \phi(x)}{\Phi(x)-\Phi(x-a)}\right)=\left(\sigma_{\theta_{i}} \beta_{j}\right)^{2} \cdot\left((\Omega(x, a))^{2}-\Delta(x, a)\right),
\end{gathered}
$$

where

$$
x=\beta_{j} \theta_{i}+\alpha_{j}-\gamma_{c-1} \quad \text { and } \quad a=\gamma_{c}-\gamma_{c-1},
$$

and $\Omega$ and $\Delta$ are as in equation (30) of the main paper,

$$
\begin{aligned}
& \Omega(x, a)=\frac{\phi(x)-\phi(x-a)}{\Phi(x)-\Phi(x-a)} \\
& \Delta(x, a)=\frac{x \phi(x)-(x-a) \phi(x-a)}{\Phi(x)-\Phi(x-a)}+\left(\frac{\phi(x)-\phi(x-a)}{\Phi(x)-\Phi(x-a)}\right)^{2} .
\end{aligned}
$$


Step 2. Plug the derivatives of $f$ into the right-hand sides of (16) and (20) of the main paper. This gives expressions for the posterior means and variances of the parameters. For instance, for $\alpha_{j}$ we have

$$
\begin{aligned}
E\left(\alpha_{j} \mid y_{i j}\right) & =\mu_{\alpha_{j}}+\sigma_{\alpha_{j}}^{2} E\left(\Omega(x, a) \mid y_{i j}\right), \\
\operatorname{Var}\left(\alpha_{j} \mid y_{i j}\right) & =\sigma_{\alpha_{j}}^{2}\left[1+\sigma_{\alpha_{j}}^{2} E\left((\Omega(x, a))^{2}-\Delta(x, a) \mid y_{i j}\right)-\sigma_{\alpha_{j}}^{2}\left(E(\Omega(x, a)) \mid y_{i j}\right)^{2}\right] .
\end{aligned}
$$

Step 3. Evaluate the expectations in Step 2. Below we show how to employ (4) and equations (6)-(7) below to derive the first equation in (26) of the main paper. To begin, we need the following results: for $c>0$,

$$
\begin{aligned}
& \int_{-\infty}^{\infty} \phi(\theta \mid \mu, \sigma) \phi(b \mid a \theta, c) d \theta=\phi\left(b \mid a \mu, \sqrt{a^{2} \sigma^{2}+c^{2}}\right)=\frac{1}{\sqrt{2 \pi\left(a^{2} \sigma^{2}+c^{2}\right)}} e^{-\frac{(a \mu-b)^{2}}{2\left(a^{2} \sigma^{2}+c^{2}\right)}}, \\
& \int_{-\infty}^{\infty} \phi(\theta \mid \mu, \sigma) \Phi\left(\frac{b-a \theta}{c}\right) d \theta=\Phi\left(\frac{b-a \theta}{\sqrt{a^{2} \sigma^{2}+c^{2}}}\right) .
\end{aligned}
$$

These equations are obtained by writing the function $\Phi(\cdot)$ as an integral and interchanging the order of the integrals. By (3), we have

$$
\begin{aligned}
& E\left(\Omega(x, a) \mid y_{i j}\right)=\iiint\left(\frac{\phi(x)-\phi(x-a)}{\Phi(x)-\Phi(x-a)}\right) p\left(\alpha_{j}, \beta_{j}, \theta_{i} \mid y_{i j}\right) d \alpha_{j} d \beta_{j} d \theta_{i} \\
= & \frac{\iiint[\phi(x)-\phi(x-a)] \phi\left(\alpha_{j} \mid \mu_{\alpha_{j}}, \sigma_{\alpha_{j}}\right) \phi\left(\beta_{j} \mid \mu_{\beta_{j}}, \sigma_{\beta_{j}}\right) \phi\left(\theta_{i} \mid \mu_{\theta_{i}}, \sigma_{\theta_{i}}\right) d \alpha_{j} d \beta_{j} d \theta_{i}}{\iiint[\Phi(x)-\Phi(x-a)] \phi\left(\alpha_{j} \mid \mu_{\alpha_{j}}, \sigma_{\alpha_{j}}\right) \phi\left(\beta_{j} \mid \mu_{\beta_{j}}, \sigma_{\beta_{j}}\right) \phi\left(\theta_{i} \mid \mu_{\theta_{i}}, \sigma_{\theta_{i}}\right) d \alpha_{j} d \beta_{j} d \theta_{i}} \\
= & \frac{I_{1}-I_{2}}{I I_{1}-I I_{2}} .
\end{aligned}
$$

For $I_{1}$, write $\phi(x)=\phi\left(\beta_{j} \theta_{i}+\alpha_{j}-\gamma_{c-1}\right)=\phi\left(\gamma_{c-1}-\beta_{j} \theta_{i} \mid \alpha_{j}, 1\right)$, apply (6) twice and use the relation $\phi(\theta \mid \mu, \sigma)=\phi(\mu \mid \theta, \sigma)$ to obtain

$$
\begin{aligned}
& I_{1}=\iiint \phi\left(\gamma_{c-1}-\beta_{j} \theta_{i} \mid \alpha_{j}, 1\right) \phi\left(\alpha_{j} \mid \mu_{\alpha_{j}}, \sigma_{\alpha_{j}}\right) \phi\left(\beta_{j} \mid \mu_{\beta_{j}}, \sigma_{\beta_{j}}\right) \phi\left(\theta_{i} \mid \mu_{\theta_{i}}, \sigma_{\theta_{i}}\right) d \alpha_{j} d \beta_{j} d \theta_{i} \\
= & \iint \phi\left(\gamma_{c-1}-\beta_{j} \theta_{i} \mid \mu_{\alpha_{j}}, \sqrt{1+\sigma_{\alpha_{j}}^{2}}\right) \phi\left(\beta_{j} \mid \mu_{\beta_{j}}, \sigma_{\beta_{j}}\right) \phi\left(\theta_{i} \mid \mu_{\theta_{i}}, \sigma_{\theta_{i}}\right) d \beta_{j} d \theta_{i} \\
= & \iint \phi\left(\gamma_{c-1}-\mu_{\alpha_{j}} \mid \beta_{j} \theta_{i}, \sqrt{1+\sigma_{\alpha_{j}}^{2}}\right) \phi\left(\beta_{j} \mid \mu_{\beta_{j}}, \sigma_{\beta_{j}}\right) \phi\left(\theta_{i} \mid \mu_{\theta_{i}}, \sigma_{\theta_{i}}\right) d \beta_{j} d \theta_{i} \\
= & \int \phi\left(\gamma_{c-1}-\mu_{\alpha_{j}} \mid \mu_{\beta_{j}} \theta_{i}, \sqrt{1+\sigma_{\alpha_{j}}^{2}+\sigma_{\beta_{j}}^{2} \theta_{i}^{2}}\right) \phi\left(\theta_{i} \mid \mu_{\theta_{i}}, \sigma_{\theta_{i}}\right) d \theta_{i} .
\end{aligned}
$$

For the last line, we can not apply (6) to evaluate the integral because $\theta_{i}$ appears in the variance of the $\phi$ function. Now assume that $\sigma_{\theta_{i}}$ is small, so that $\theta_{i}$ is close to $\mu_{\theta_{i}}$ with a high probability. In our experiments, $\sigma_{\theta_{i}}$ is smaller than 1 . So, we approximate $\theta_{i}$ in the variance by $\mu_{\theta_{i}}$ to obtain (8), and then apply (6) to obtain (9):

$$
\begin{aligned}
I_{1} & \approx \int \phi\left(\gamma_{c-1}-\mu_{\alpha_{j}} \mid \mu_{\beta_{j}} \theta_{i}, \sqrt{1+\sigma_{\alpha_{j}}^{2}+\sigma_{\beta_{j}}^{2} \mu_{\theta_{i}}^{2}}\right) \phi\left(\theta_{i} \mid \mu_{\theta_{i}}, \sigma_{\theta_{i}}\right) d \theta_{i} \\
& =\phi\left(\gamma_{c-1}-\mu_{\alpha_{j}} \mid \mu_{\beta_{j}} \mu_{\theta_{i}}, \sqrt{1+\sigma_{\alpha_{j}}^{2}+\sigma_{\beta_{j}}^{2} \mu_{\theta_{i}}^{2}+\sigma_{\theta_{i}}^{2} \mu_{\beta_{j}}^{2}}\right) \\
& =\frac{1}{\nu} \phi\left(\frac{\mu_{\beta_{j}} \mu_{\theta_{i}}+\mu_{\alpha_{j}}-\gamma_{c-1}}{\nu}\right),
\end{aligned}
$$


where $\nu=\left(1+\sigma_{\alpha_{j}}^{2}+\sigma_{\beta_{j}}^{2} \mu_{\theta_{i}}^{2}+\sigma_{\theta_{i}}^{2} \mu_{\beta_{j}}^{2}\right)^{1 / 2}$. The expression for $I_{2}$ is the same as $I_{1}$ except that $\gamma_{c-1}$ is replaced by $\gamma_{c}$. The calculations of $I I_{1}$ and $I I_{2}$ are similar, but will use (7) rather than (6); we omit the details and only present the results:

$$
I I_{1} \approx \Phi\left(\frac{\mu_{\theta_{i}} \mu_{\beta_{j}}+\mu_{\alpha_{j}}-\gamma_{c-1}}{\nu}\right), \quad I I_{2} \approx \Phi\left(\frac{\mu_{\theta_{i}} \mu_{\beta_{j}}+\mu_{\alpha_{j}}-\gamma_{c}}{\nu}\right) .
$$

Therefore, together with (4), the posterior mean of $\alpha_{j}$ can be approximated by

$$
\tilde{\mu}_{\alpha_{j}}=\mu_{\alpha_{j}}+\frac{\sigma_{\alpha_{j}}^{2}}{\nu} \frac{\phi\left(\frac{\mu_{\theta_{i}} \mu_{\beta_{j}}+\mu_{\alpha_{j}}-\gamma_{c-1}}{\nu}\right)-\phi\left(\frac{\mu_{\theta_{i}} \mu_{\beta_{j}}+\mu_{\alpha_{j}}-\gamma_{c}}{\nu}\right)}{\Phi\left(\frac{\mu_{\theta_{i}} \mu_{\beta_{j}}+\mu_{\alpha_{j}}-\gamma_{c-1}}{\nu}\right)-\Phi\left(\frac{\mu_{\theta_{i}} \mu_{\beta_{j}}+\mu_{\alpha_{j}}-\gamma_{c}}{\nu}\right)} .
$$

This establishes the first equation in (26) of the main paper. The second equation in (26) can be derived analogously by employing (5)-(7). The formulas in (27) and (28) of the main paper can be obtained similarly and we omit the derivations.

\section{Appendix B}

Here we give the proof of Lemma 1 in the main paper.

Proof. We only prove (a). The proof of (b) is similar and is omitted; (c) is a simple consequence of (a) and (b). Part (d) can be derived by L'Hôpital's rule. For (a), we have

$$
\begin{aligned}
\Lambda\left(\frac{a}{2}+z, a\right) & =\frac{\left(\frac{a}{2}+z\right) \phi\left(\frac{a}{2}+z\right)-\left(z-\frac{a}{2}\right) \phi\left(z-\frac{a}{2}\right)}{\Phi\left(\frac{a}{2}+z\right)-\Phi\left(z-\frac{a}{2}\right)} \\
& =\frac{\left(\frac{a}{2}+z\right) \phi\left(-\frac{a}{2}-z\right)-\left(z-\frac{a}{2}\right) \phi\left(\frac{a}{2}-z\right)}{1-\Phi\left(-\frac{a}{2}-z\right)-1+\Phi\left(\frac{a}{2}-z\right)} \\
& =\frac{\left(\frac{a}{2}-z\right) \phi\left(\frac{a}{2}-z\right)-\left(-\frac{a}{2}-z\right) \phi\left(-\frac{a}{2}-z\right)}{\Phi\left(\frac{a}{2}-z\right)-\Phi\left(-\frac{a}{2}-z\right)} \\
& =\Lambda\left(\frac{a}{2}-z, a\right),
\end{aligned}
$$

where the second equality follows from $\phi(x)=\phi(-x)$ and $\Phi(x)=1-\Phi(-x)$. 